Hier können Sie CME-Punkte sammeln a) für die Pflichtfortbildung aller Vertragsärzte und b) für freiwillige Fortbildungszertifikate, die viele Landesärztekammern anbieten.

Die Multiple-Choice-Fragen beziehen sich auf den vorangegangenen Fortbildungsbeitrag (Seiten 25-29). Die Antworten ergeben sich z. T. aus dem Text, z. T. beruhen sie auf medizinischem Basiswissen!
CME-Herausgeber- und Review-Board: Dr. H. J. K. Barwitz, Prof. Dr. A. Berghaus, Prof. Dr. Dr. h.c. Th. Brandt, Prof. Dr. W. G. Daniel, Prof. Dr. W. Eisenmenger, Prof. Dr. K. Friese, Prof. Dr. H. S. Füessl, Prof. Dr. B. Göke, Prof. Dr. R. Gradinger, Prof. Dr. H. Holzgreve, Prof. Dr. A. Imdahl, Prof Dr. K.-W. Jauch, Prof. Dr. K. Krüger, Prof. Dr. H.-J. Möller, Prof. Dr. D. Reinhardt, Prof. Dr. Dr. h.c. Th. Ruzicka, Prof. Dr. Ch. Stief, U. Weigeldt.

\title{
Fragen zum Thema „Schilddrüsenknoten aus endokrinologischer Sicht"
}

\section{Wie ist die Prävalenz von Strumen und} Schilddrüsenknoten in Deutschland?

A Zunehmend

B Abnehmend

C Bei 20-24-Jährigen > 10\%

D Unverändert

E Nicht ausreichend evaluiert

\section{Welches Untersuchungsverfahren dient der Abklärung von heißen und kalten Knoten?}

A Sonografie

B Palpation

C Szintigrafie

D Doppler-Sonografie

E Computertomografie

\section{- 3. Frühe Symptome eines} Schilddrüsenkarzinoms ist/sind

A Schluckbeschwerden.

B ein tastbarer Knoten.

C vergrößerte Halslymphknoten.

D Heiserkeit.

E keines von A-D.

\footnotetext{
- 4. Ein erhöhtes Malignomrisiko bei Schilddrüsenknoten besteht bei

A Immunthyreoiditis.

B akut-subakuter Thyreoiditis.

C funktioneller Schilddrüsenautonomie.

D Strahlenexposition in der Kindheit.

E Schilddrüsenzysten.
}

\author{
- 5. Welcher der nachfolgenden Befunde \\ ist besonders typisch beim medullären \\ Schilddrüsenkarzinom? \\ A Unscharfe Randbegrenzung \\ B Mikrokalk \\ C Echoreicher Knoten \\ D Ringperfusion \\ E Größe $>5 \mathrm{~cm}$
}
-6. Welches der folgenden Merkmale macht den Halslymphknoten malignom- verdächtig?
A Größe $>1 \mathrm{~cm}$
B Runde Form (Länge/Dicke $<2 \mathrm{~cm}$ )
C Gefäßhilus
D Randperfusion
E Echoarmut

7. Mit welchem Laborbefund Iäs
eine Schilddrüsenfunktionsstö
hoher Sicherheit ausschließen
stätigen?
A TSH-Rezeptor-Antikörper
B Thyreoglobulin-Antikörper
C Calcitonin-Bestimmung
D TSH
E fT4

- 8. Welcher Tumormarker ist beim Schilddrüsenkarzinom tumorspezifisch?

A Thyreoglobulin

B CEA

C Calcitonin

D Thyroxinbindendes Globulin

E Schilddrüsenantikörper

- 9. Welcher Schilddrüsenknoten sollte dringend operiert werden?

A Echoreicher, kühler Knoten von 1,5 $\mathrm{cm}$ mit Randvaskularisation

B $2 \mathrm{~cm}$ großer, zystisch degenerierter Knoten, punktionszytologisch unverdächtig

C 20-jähriger Patient mit echoarmem Knoten und Wachstumstendenz $>20 \%$ in sechs Monaten

D Patient mit Hashimoto-Thyreoiditis und mehreren $1 \mathrm{~cm}$ großen Knoten

E Unifokale Autonomie ohne Lokalsymptome mit TSH 0,3 mU/1

- 10. Wann ist die Indikation zur Schilddrüsenszintigrafie gegeben?

A Bei Knoten $>1,5 \mathrm{~cm}$

B Bei diffuser Struma zur Beurteilung der Jodavidität

C Bei echoreichen Knoten um $1 \mathrm{~cm}$

D Bei Schilddrüsenzysten

E Zum Beweis eines malignen Knotens

\section{ANTWORTFORMULAR}

Mit Einreichen dieses Fragebogens erkläre ich mich damit einverstanden, dass die angegebenen Daten zum Zweck der Teilnahmebestätigung gespeichert und bei erfolgreicher Teilnahme an den Einheitlichen Informationsverteiler (EIV) der Ärztekammern weitergegeben werden.

Wichtiger Hinweis:

Eine Auswertung ist künftig nur noch möglich, wenn Sie Ihre EFN auf dem Antwortformular angeben!

Nähere Hinweise hierzu unter: www.cme-punkt.de/faq.htm

\section{"Schilddrüsenknoten aus endokrinologischer Sicht"}

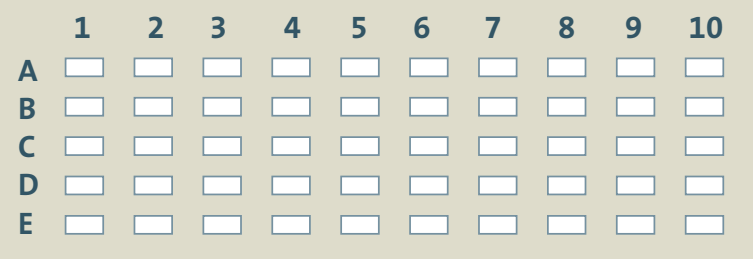

Bitte kreuzen Sie pro Frage je eine Antwort deutlich an.

\begin{tabular}{ll}
\hline Name, Vorname & Fachrichtung \\
\hline Geburtsdatum & Einheitliche Fortbildungsnummer (EFN) \\
\hline Straße, Nr. & PLZ, Ort- \\
\hline Ich versichere, alle Fragen ohne fremde & Unterschrift
\end{tabular}

Und so kommen Sie zu Ihren Punkten:

Teilnahme im Internet: unter www.cme-punkt.de. Dort führen wir für Sie ein elektronisches Punktekonto.

Teilnahme per Brief: Fragebogen ausfüllen und mit frankiertem Rückumschlag an: Urban \& Vogel GmbH CME MMW-Fortschr. Med. Postfach 81664 München. 